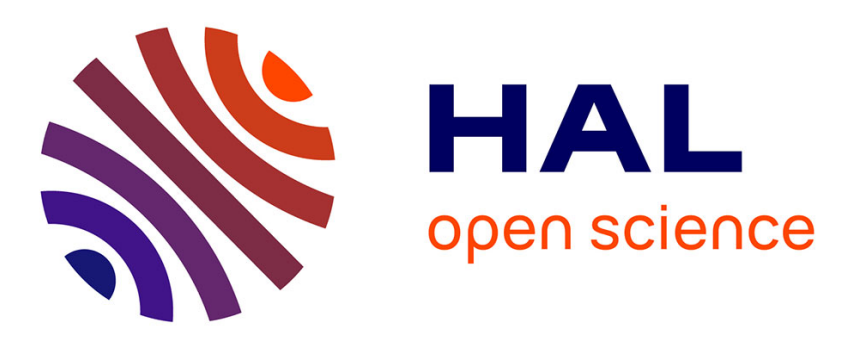

\title{
An experimental XAS and ab initio approach to describe the electronic and local structure of sodium nitroprussiate single crystals
}

Duclair Tchana Kamgne, Bridinette Thiodjio Sendja, Danilo Oliveira de Souza, Dominik Schaniel, Giuliana Aquilanti

\section{To cite this version:}

Duclair Tchana Kamgne, Bridinette Thiodjio Sendja, Danilo Oliveira de Souza, Dominik Schaniel, Giuliana Aquilanti. An experimental XAS and ab initio approach to describe the electronic and local structure of sodium nitroprussiate single crystals. Journal of Molecular Structure, 2021, 1245, pp.131119. 10.1016/j.molstruc.2021.131119 . hal-03401215

\section{HAL Id: hal-03401215 \\ https://hal.univ-lorraine.fr/hal-03401215}

Submitted on 25 Oct 2021

HAL is a multi-disciplinary open access archive for the deposit and dissemination of scientific research documents, whether they are published or not. The documents may come from teaching and research institutions in France or abroad, or from public or private research centers.
L'archive ouverte pluridisciplinaire HAL, est destinée au dépôt et à la diffusion de documents scientifiques de niveau recherche, publiés ou non, émanant des établissements d'enseignement et de recherche français ou étrangers, des laboratoires publics ou privés. 


\title{
An experimental XAS and $a b$ initio approach to describe the electronic and local structure
}

\section{of sodium nitroprussiate single crystals}

Duclair Tchana Kamgne ${ }^{1}$, Bridinette Thiodjio Sendja ${ }^{2}$, Danilo Oliveira de Souza ${ }^{3 *}$, Dominik

Schaniel $^{4}$, Giuliana Aquilanti ${ }^{3}$

${ }^{1}$ University of Yaounde I, Faculty of Science, Department of Physics, Yaounde, Cameroon

${ }^{2}$ University of Yaounde I, National Advanced School of Engineering, Department of

Mathematic and Physical Science, Yaounde, Cameroon

3 Elettra-Sincrotrone Trieste, s.s. 14 - km 163,5 in AREA Science Park. 34149 Basovizza, Trieste ITALY

${ }^{4}$ University of Lorraine, CNRS, CRM2, Nancy, France

*Corresponding Author: E-mail: danilo.oliveiradesouza@elettra.eu

\begin{abstract}
The electronic and local structural properties of single crystals in the ground state (GS) of the photo-switchable compound $\mathrm{Na}_{2}\left[\mathrm{Fe}(\mathrm{CN})_{5} \mathrm{NO}\right] \cdot 2 \mathrm{H}_{2} \mathrm{O}$ (sodium nitroprussiate, $\mathrm{SNP}$ ) have been investigated by X-ray Absorption Fine Structure Spectroscopy (XAFS) and FEFF simulations. In SNP, the XANES (X-ray Absorption Near Edge Structure) pre-edge region presents three distinct peaks with different intensities depending on the crystal axis orientation with respect to the polarization direction of the X-ray beam. The peaks correspond to a combination of $1 \mathrm{~s}$ to $4 \mathrm{p}$ and $1 \mathrm{~s}$ to $3 \mathrm{~d}$ transitions with possibly mixed dipole and quadrupole character. The EXAFS fitting reveals, as expected, a biased structure according to the relative position of the crystal with respect to the beam polarization and the fittings are in accordance to theoretical values. The best fitted model has a large contribution from multi-scattering paths, which clarifies the interpretation of the actual peak contributions on the Fourier Transformed signal.
\end{abstract}

Keywords: Photoswitchable compound ; Electronic properties ; X-ray Absorption Spectroscopy ; Pre-edge features ; NO ligand

\section{Introduction}

$\mathrm{Na}_{2}\left[\mathrm{Fe}(\mathrm{CN})_{5} \mathrm{NO}\right] \cdot 2 \mathrm{H}_{2} \mathrm{O}$ or sodium nitroprussiate (SNP) is an intriguing compound with interesting properties. On the one hand, it is well known and used in clinical applications, namely as a vasodilator [1] but has also been investigated for cancer therapeutics due to its potential as a nitric oxide donor [2]. On the other hand, SNP has been investigated due to its photosensitivity, especially since the discovery of so-called metastable states (see e.g. [3] for a review of these properties). As a matter of fact, these metastable states correspond to linkage isomers of the nitrosyl ligand, which are stable at low temperatures (at $100 \mathrm{~K}$ the lifetime is several years). As shown by X-ray and neutron diffraction as well as DFT calculations, the NO ligand can adopt three different structural configurations with respect to the central iron atom 
of the $\left[\mathrm{Fe}(\mathrm{CN})_{5} \mathrm{NO}\right]^{2-}$ octahedron: the nitrogen bound linear $\mathrm{Fe}-\mathrm{N}-\mathrm{O}$ ground state $(\mathrm{GS})$, the oxygen bound linear Fe-O-N metastable state 1 (MS1) and the side-on bound bent $\eta^{2}$ (FeNO) metastable state 2 (MS2) [4], [5], [6], [7], [8]. The generation of these photoinduced linkage isomers is ultrafast (a few hundred femtoseconds) and has been studied by time-resolved optical and infrared spectroscopy on single crystals $[\mathbf{9 , 1 0 ]}$ and is accompanied by large photochromic and photorefractive effects $[11,12]$. These different structural configurations can be reversibly transferred between GS, MS1, and MS2 depending on the chosen irradiation wavelength as well as the polarization direction of the light with respect to the crystal axes [13]. The peculiar polarization dependent properties of SNP have been investigated by polarized optical absorption spectroscopy on single crystals which revealed the light-induced absorption changes in SNP caused by the excitation of MS1 and MS2 and allowed for the assignment of the electronic transitions and comparison with theoretical results [11]. Overall, to understand the properties of the metastable and ground states in SNP, many different techniques have been employed, but only a few studies using X-ray Absorption Spectroscopy (XAS), namely polarization dependent XAS on SNP single crystals on the Fe K-edge [14] and XAS on the Fe L2,3 edge on SNP powder [15]. Gadeke et al (1988) [14] reported a strong dependence of the XANES and EXAFS spectra on the angle between polarization direction of the X-ray beam and the crystal axis.

XAS is element-specific and is suited to study in detail the local environment (geometry and electronic configuration) of a given atom to gain accurate insight into small changes that would occur upon photoexcitation of linkage isomers such as MS1 or MS2 in SNP. In this work we report a XAS experimental and ab initio study on the GS of SNP single crystals in different crystal axis orientation with a full modelling of the EXAFS and XANES spectra, which allow us to get newer insight on the fine structure of the molecule and pave the path to tailored in situ experiments in order to fully understand the physics behind metastable states on this compound. XAS is able to determine around the photo-absorber the interatomic distance, the number and type of species of the neighboring atoms, and the thermal or structural disorder of the positions of the neighboring atoms. It is a powerful tool which can provide chemical shortrange structural and electronic information of the neighbourhood of the photo absorber in various materials. It has been extensively used for such complex materials [[16], [17], [18], [19], [20]].

\section{Materials and Methods}

\subsection{Sample preparation}

Large single crystals of SNP were recrystallized by slow evaporation from aqueous solutions using reagent grade Sodium nitroprusside dehydrate $\mathrm{Na}_{2}\left[\mathrm{Fe}(\mathrm{CN})_{5} \mathrm{NO}\right] \cdot 2 \mathrm{H}_{2} \mathrm{O}$. Thin plates of the orthorhombic crystals (space group Pnnm [21,22]) were cut perpendicular to the crystal axes a, b, or c and ground to thicknesses of about $100 \mu \mathrm{m}$ before being polished to optical quality. Powder of SNP was produced by grinding single crystals.

\subsection{XAS experimental details}

The SNP samples used for measurements were a powder and crystals cut along the, a-, band c- crystal axis. The ideal thickness of SNP for XAS measurements in transmission mode, 
is $\sim 100 \mu \mathrm{m}$ yielding an absorption jump of 1.2 and a total absorption of 1.55 . These values are calculated considering the Fe K edge. Three different SNP crystals were used for the XAS measurements, corresponding to cuts perpendicular to the, a-, b-, and c-axis. The three platelike crystals had a thickness of about $84 \mu \mathrm{m}$ along a- and c-axis, and $165 \mu \mathrm{m}$ along b-axis. The lateral dimensions of the crystals were $10 \mathrm{~mm} \times 3 \mathrm{~mm}$. The SNP powder was mixed with polyvinylpyrrolidone (PVP) in a proper dilution to have an absorption jump of $\sim 1$ and then compacted in a pellet of $13 \mathrm{~mm}$ of diameter. XAS measurements were carried out in transmission mode for powder and crystal samples at room temperature, at the Fe K edge (7112 $\mathrm{eV}$ ) at the XAFS beamline [23] of Elettra Synchrotron (Trieste, Italy). The storage ring was operated at $2 \mathrm{GeV}$ yielding a minimum current of $309 \mathrm{~mA}$. A double crystal silicon Si (111) monochromator was used with a detuning of 0.3 for harmonic rejection and the energy resolution $\Delta \mathrm{E} / \mathrm{E}$ was about $10^{-4}$. The value of $7112 \mathrm{eV}$ was assigned to the first inflexion point of the XANES spectrum of Fe metal foil for the calibration of the monochromator. The ions chambers were filled with a mixture of $\mathrm{Ar}, \mathrm{N}_{2}$ and $\mathrm{He}$ to have an absorption of $10 \%, 80 \%$ and $95 \%$ in the $\mathrm{I}_{0}, \mathrm{I}_{1}$, and $\mathrm{I}_{2}$ respectively at the energy of $7000 \mathrm{eV}$. The data were collected in the energy range of [6817-8350] eV with an energy step variable depending on the energy region. At least two scans were collected for the powder sample and four for crystals. The crystals were placed such as a-, b- or c- axis was perpendicular to the electric field $\vec{E}$ of the X-ray incident beam characterized by a wavevector $\vec{k}$ parallel to the crystal face considered.

\subsection{EXAFS data analysis}

EXAFS data analysis was performed for all measurements using the least-square fitting based on the multiple scattering approximation. The normalization and background extraction steps were done in Athena program according to Ravel and Newville [24]. Spectra of the same sample were averaged in order to improve the statistical noise. Data were then normalized to one absorption event and aligned within $0.01 \mathrm{eV}$. The EXAFS signal was obtained as shown in the following equation:

$$
\chi(\mathbf{E})=\frac{\left(\mu-\mu_{0}\right)}{\mu_{0}} .
$$

Where $\mu$ and $\mu_{0}$ are the experimental absorption coefficient and a smooth spline representing the embedded-atom absorption background respectively. This step was followed by the Fourier Transform (FT) of the k-weighted EXAFS functions $k^{2} \chi(E)$ using a Hanning window in the $\mathrm{k}$ range of [3-14] $\AA^{-1}$. In order to evaluate the backscattering amplitudes and the phase shifts parameters, the FEFF6 code based on the ab initio multiple scattering calculations [24] was used to generate the theoretical EXAFS signals using a cif file with the crystallographic parameters taken from Carducci et al. [5]. The fitting of the theoretical signals on the EXAFS experimental data was performed using Artemis program [24]. The data were fitted considering the $k$ range of [3-14] $\AA^{-1}$ and $R$ range of [1.1-3.0], [1.3-3.0] $\AA$ (respectively, for c-axis and aaxis). The fit for the powder were performed in the $k$ range of [3-15.5] $\AA^{-1}$ and $R$ of [1.1-3.0] $\AA$. The reliability of the fits is reported through the $R_{\text {factor }}$ parameter minimizing the difference between the experimental $\left(\boldsymbol{k}_{\boldsymbol{s}}^{2} \chi\left(\boldsymbol{k}_{\boldsymbol{s}}\right)_{\text {exp }}\right)$ and calculated $\left(\boldsymbol{k}_{\boldsymbol{s}}^{2} \chi\left(\boldsymbol{k}_{\boldsymbol{s}}\right)_{\text {calc }}\right)$ spectra according to the following equation $[\mathbf{2 5 , 2 6 ]}$

$$
R_{\text {factor }}=\sum_{s} \frac{\left|k_{s}^{2} \chi\left(k_{s}\right)_{\exp }-k_{s}^{2} \chi\left(k_{s}\right)_{\text {calc }}\right|}{\left|k_{S}^{2} \chi\left(k_{s}\right)_{\exp }\right|} .
$$




\subsection{XAS simulation}

$A b$ initio XANES simulations were obtained by performing full multiple scattering calculations using FEFF9.0 at the Fe K edge of SNP based on the crystallographic structure obtained by Carducci et al. [5]. The XANES simulation in FEFF9 considers the treatment of the core-hole within the random phase approximation (RPA), the many-pole self-energy and the many-body spectral function convolution [27]. We have used a self-consistent energy dependent exchange correlation Hedin-Lunqvist potential [28]. Self-consistency was obtained by successively calculating the electron density of states, electron density and Fermi level at each stage of simulation within a cluster centered on Fe atom of $3.3 \AA$, and then iterating. Full multiple scattering (FMS) calculations were carried out for a larger cluster of $4.6 \AA$. To simulate different orientations of the crystal as supposed to be irradiated by the linearly polarized x-ray beam, the polarization and ellipticity were used and adjusted accordingly. Further details on XANES simulations are available as Supplementary Material (SI).

The EXAFS simulation was done in linear polarization. It was performed through the calculations of potentials and phase shifts for a distance up to $5 \AA$. The paths with a represented weight percentage characterizing the scattering from the nearest neighbors were considered and the number was limited to improve the EXAFS results. The simulation was satisfactorily done in the Debye correlated model with the Debye-Waller factor adjusted to $0.02 \AA^{2}$ and Debye-temperature range of [190-315] K.

\section{Results and discussion}

\subsection{Crystal orientation}

Synchrotron radiation is linearly polarized in the orbit plane. Given that the wavevector $\vec{k}$ is perpendicular to the electric field of the $\mathrm{x}$-ray beam, the orientation of the crystal is given with respect to the polarized $\mathrm{x}$-ray beam that is characterized by the wavevector $\vec{k}$ and therefore the electric field $\vec{E}$. Fig. 1 presents the main aspects of the crystal structure of SNP and the orientation of the $\left[\mathrm{Fe}(\mathrm{CN})_{5} \mathrm{NO}\right]^{2-}$ anion with respect to the crystal axes. In particular we note that the quasi-fourfold axis C-N-Fe-N-O lies within the ab-plane (Fe-N-O angle of $176.2^{\circ}$, CFe-N angle of $176.8^{\circ}$, and Fe-C-N angle of $179.8^{\circ}$ ). In the case of $\vec{k} / / \vec{a}$, the electric field $\vec{E}$ can be parallel either to b- or to c- crystal axis [29]. In the same way, for $\vec{k} / / \vec{b}, \vec{E}$ can be parallel to a- or c- crystal axis while it can be parallel to a- or b- crystal axis in the case of $\vec{k} / / \vec{c}$. By reasoning so, six orientations can be obtained. Fig. 1 illustrates the situation for a $b$ cut crystal, for which the propagation vector $\vec{k} / / \vec{b}$ offers two possibilities to orient the electric field vector $\vec{E}$ along the a- or c-axis of the crystal. If $\vec{E}$ is along the c-axis, the electric field oscillates thus perpendicular to the $\mathrm{C}-\mathrm{N}-\mathrm{Fe}-\mathrm{N}-\mathrm{O}$ axis, whereas in the case of $\vec{E}$ is along the aaxis, the electric field vector oscillates along a and has thus a component parallel to $\mathrm{C}-\mathrm{N}-\mathrm{Fe}-$ $\mathrm{N}-\mathrm{O}$ axis. 


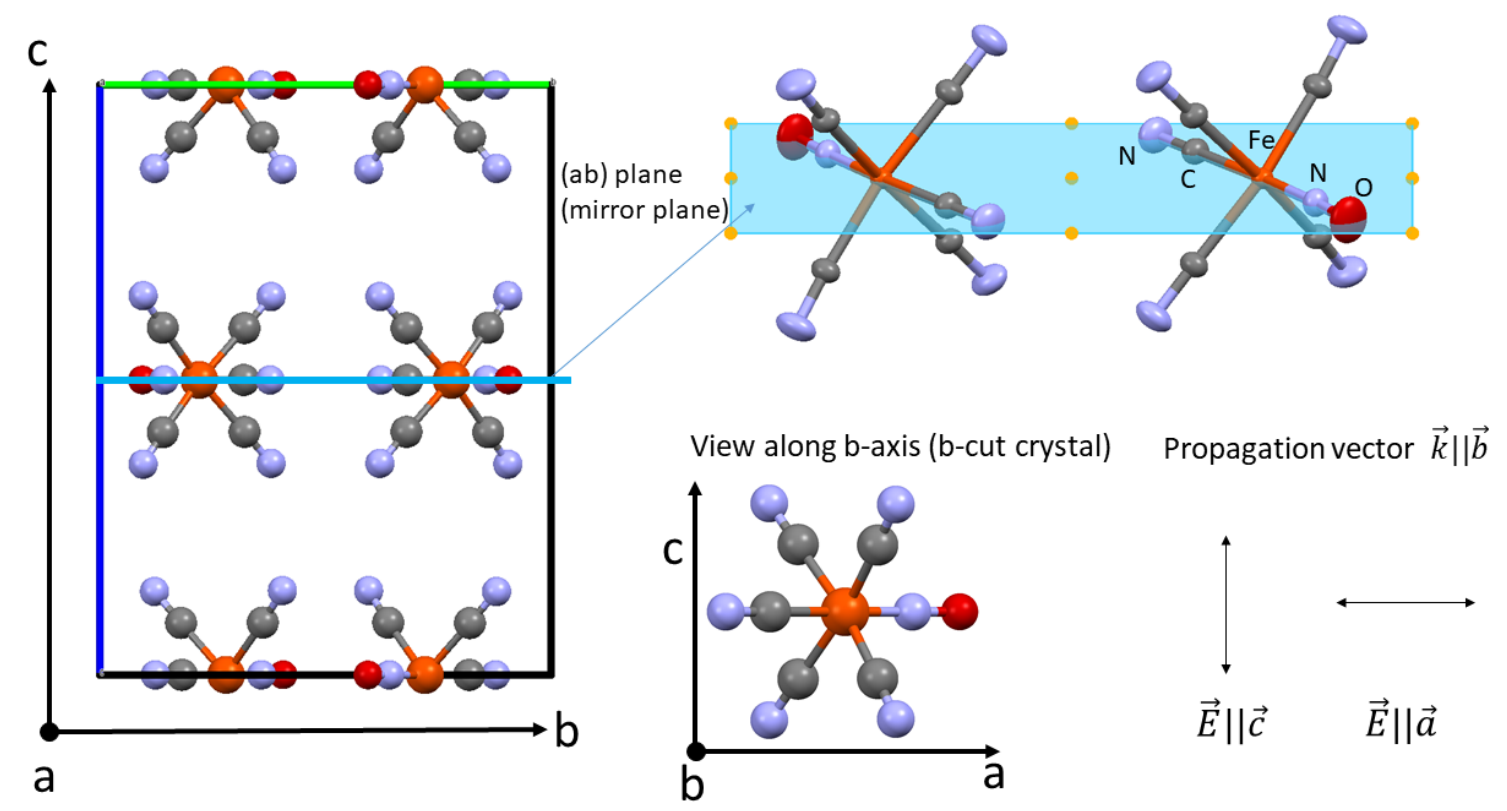

Figure 1: (Left and top panel) Representation of the crystal structure of SNP (view along aaxis, sodium counter ions and water molecules have been omitted for clarity). The quasifourfold axis $\mathrm{C}-\mathrm{N}-\mathrm{Fe}-\mathrm{N}-\mathrm{O}$ lies within the ab-plane. (Lower right panel) If the b-axis of the crystal is parallel to the propagation vector of the X-ray beam $(\vec{k} / / \vec{b})$, the electric field vector can be oriented $\vec{E} / / \vec{a}$ ) or $\vec{E} / / \vec{c}$ ) and thus parallel or perpendicular to the C-N-Fe-N-O molecular axis.

\subsection{XANES results and discussions}

Fig. 2 presents the simulated (panel a) and experimental (panel b) XANES spectra at Fe K-edge. The experimental spectrum collected of the powder sample could be simulated without considering the polarization in FEFF9, while the polarization has been taken into account as discussed in section 2.4 to simulate the data collected on the single crystals. The experimental data show similar features although some differences can be observed. The main resonance of the spectrum with polarization parallel to the c-axis is more intense and shifted to lower energy with respect to the other polarizations, while the spectrum of the powder sample clearly presents average features. The three vertical lines highlight the pre-edge features, indicated as A, B and C. These are all well-defined for the spectrum with $\vec{E} / / \vec{c}$. The features $\mathrm{B}$ and $\mathrm{C}$ broaden significantly for the other polarization configurations, resulting in an almost complete vanishing of peak $\mathrm{C}$. 


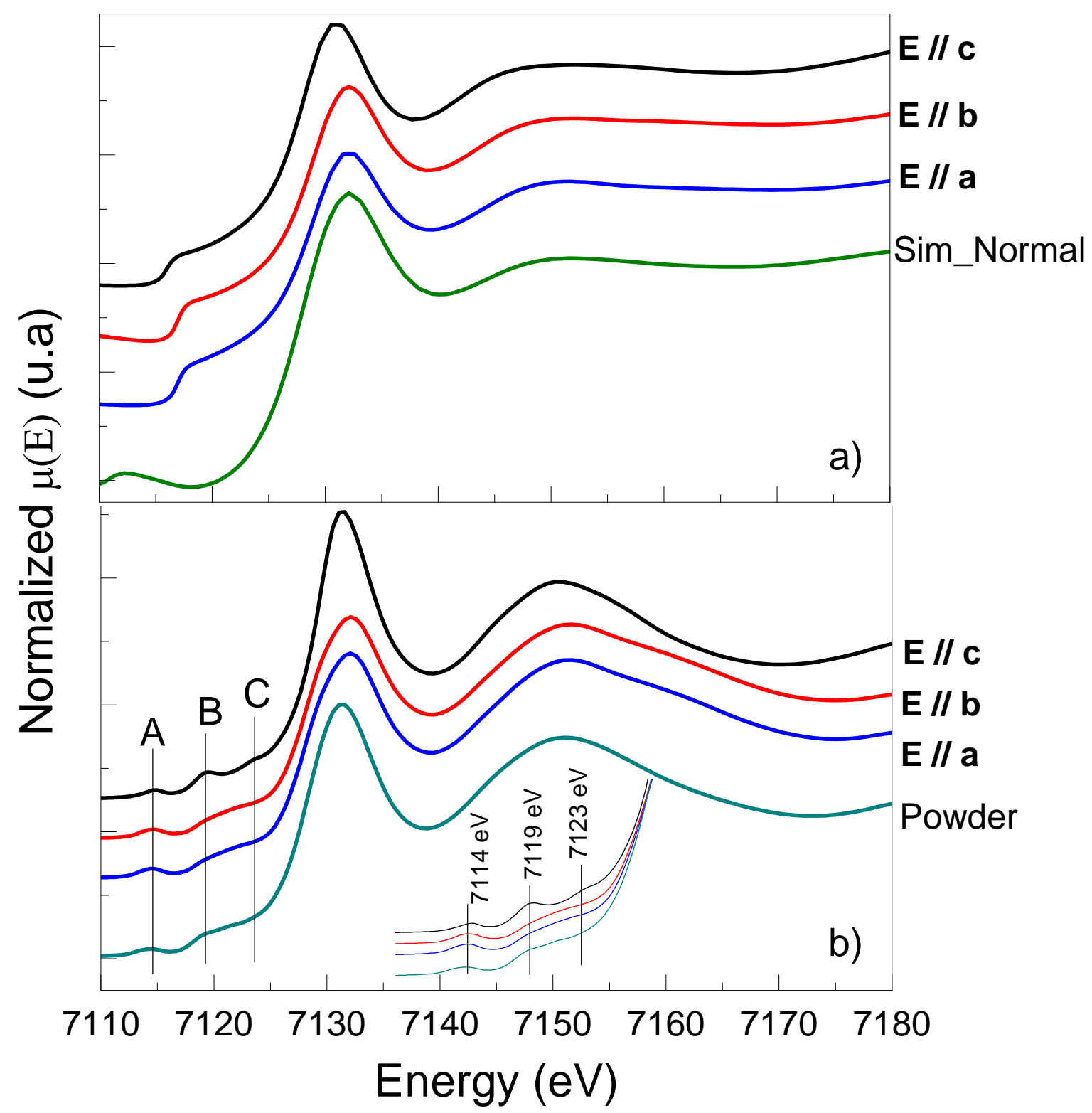

Figure 2: Normalized Fe K-edge XANES spectra of SNP samples; a) simulated data, and Sim_Normal stands for simulated data without considering a polarization factor. b) Experimental data corresponding to $\vec{E} / / \vec{a}, \vec{E} / / \vec{b}$ and $\vec{E} / / \vec{c}$. Powder stands for the data collected on the powder sample. The insert is shown to highlight the pre-edge features in the experimental spectra.

In general, the pre-peak features observed in the pre-edge region of the Fe K-edge XANES absorption and the features above the edge are characteristic of the transition to bound states $1 s \rightarrow 3 d$ and/or $1 s \rightarrow 4 p[\mathbf{3 0}, \mathbf{3 1}]$. The region above the edge generally indicates the multiple scattering diffusion and the edge-crest describes the transitions to continuum or delocalized states as reported [31]. The occupations of the $3 d$-orbitals depend on the properties of the ligands in the metal-ligand complexes. In such compounds, the molecular orbitals are formed as a linear combination between the metal valence orbitals which can be $3 \mathrm{~d}, 4 \mathrm{~s}$ and $4 \mathrm{p}$ on the one hand, and the ligand orbitals which are $\sigma, \pi, \sigma *$ and $\pi *$, on the other hand. Also, the 
interactions between them (valence-ligand) depend on the symmetry of the complexes. Thus, it can be understood that, the SNP samples corresponding to $\vec{E} / / \vec{a}, \vec{E} / / \vec{b}$ and $\vec{E} / / \vec{c}$ contain in the pre-edge region transition to hybridized orbitals with likely both, dipolar and quadrupolar characters, as manifest by the three peaks at energy values of $7114.40 \mathrm{eV}, 7119.12 \mathrm{eV}$ and $7123.76 \mathrm{eV}$.

The octahedral systems are expected to have a $\mathrm{K}$ pre-edge structure dominated by quadrupolar transitions and can be deconvoluted in distinct contributions representing, essentially, the transitions to splitted- $3 d$ components $t_{2 g}$ and $e_{g}$. However, in lower symmetries and particularly on SNP single crystals, further orbital splitting may rise, including dipolar transitions, as observed in $\mathrm{Fe}^{\mathrm{II}}$ mineral samples [32] and $\alpha-\mathrm{Fe}_{2} \mathrm{O}_{3}$ [33]. For that reason, we performed the modeling and fitting of the pre-edge region (the peak A in Fig. 2) using 2-4 pseudo-Voigt peaks. Fraction and sigma parameters of the pseudo-Voigt functions were constrained to be the same for all the peaks in the fit.

In the $\left[\mathrm{Fe}(\mathrm{CN})_{5} \mathrm{NO}\right]^{2-}$ anion of SNP the $\mathrm{C}_{4 \mathrm{v}}$ symmetry is not perfect, the $\mathrm{Fe}-\mathrm{N}-\mathrm{O}$ angle is slightly smaller than 180 degrees. However, the quasi-fourfold axis $\mathrm{N}-\mathrm{C}-\mathrm{Fe}-\mathrm{N}-\mathrm{O}$ lies in the ab plane of the orthorhombic crystal structure, giving rise to a pronounced polarization dependence of the absorption spectra. Therefore $\vec{E} / / \vec{c}$ indicates a polarization perpendicular to this rotation axis, while for $\vec{E} / / \overrightarrow{a, b}$, the polarization has contributions parallel to this fourfold rotation axis (see Fig. 1).

Considering that XANES from spectra $\vec{E} / / \vec{a}$ are equal to $\vec{E} / / \vec{b}$, we discuss them as one single representative direction. In this sense, both different crystal orientations (i.e., $\vec{E} / / \vec{a}$ and $\vec{E} / / \vec{c}$ ) were best fitted by using a 3-component model (Fig. 3), featuring a prominent one at higher energy, and intermediate intensity peak in the middle and a tiny shoulder at lower energy. Qualitatively, we observe that the individual components hold their position no matter the crystal orientation. Further, the centroid shift observed when comparing $\vec{E} / / \vec{a}$ and $\vec{E} / / \vec{c}$ is due exclusively to the increasing in amplitude of the component centered around $7113 \mathrm{eV}$, while the main component $(\sim 7114.5 \mathrm{eV})$ remains with the same height. This isotropic behavior of the latter may suggest a dipolar transition, but a dedicated experiment is required to deal with this issue. Thus, a deep analysis on the angular dependence of the pre-peak intensities will be presented in a dedicated paper. Table 1 summarizes the results of the fits. 


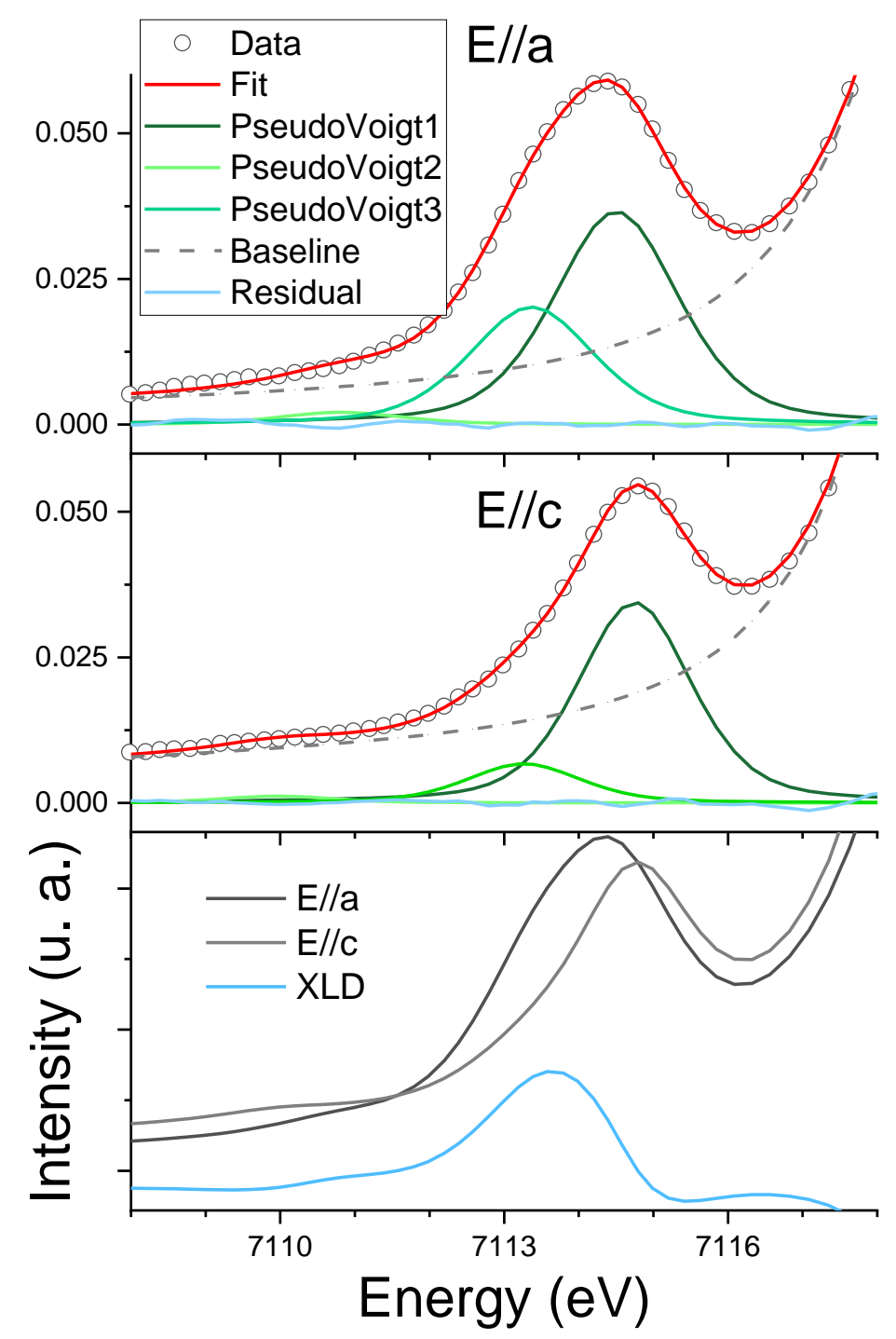

Figure 3: Fit of the pre-peak of Fe K-edge showing a deconvolution of three components related to dipolar and quadrupolar transitions. Fit corresponding to a-axis (upper panel), c-axis (middle) and experimental curves for a- and c-axis showing a clear shift in the centroid position (bottom). The blue line on the bottom panel is the difference between both experimental lines.

The energy difference between the higher-energy peak component and the lower one is $3.7 \mathrm{eV}$ and $4.8 \mathrm{eV}$, respectively, for $\vec{E} / / \vec{a}$ and $\vec{E} / / \overrightarrow{\mathrm{c}}$, which suggests a ferric low-spin electronic configuration with an increased $10 \mathrm{Dq}$ due to stronger field induced by $\mathrm{CN}^{-}$ligands, as already observed on $\mathrm{Fe}^{\mathrm{III}}$ complexes [34]. This difference is a direct probe of the $10 \mathrm{Dq}$ parameter of the crystalline field and can help us to understand the electronic structure of the crystal. In order to do that, we consider the optical absorption study on the same material performed by one of the authors to correlate to the information obtained from pre-peaks and try to get newer insights on the electronic structure of the SNP molecule. 
Table 1 - Main parameters fitted on deconvolution of Fe K-edge pre-peak of SNP. Two different ranges in energy were proposed, respectively, i) a 3-peak model ranged at 7108-7118 $\mathrm{eV}$ and ii) a 5-peak model ranged at $7108-7125 \mathrm{eV}$.

\begin{tabular}{|c|c|c|c|c|}
\hline $\begin{array}{c}\text { Crystal } \\
\text { Orientation }\end{array}$ & Peak Energy (eV) & $\begin{array}{c}\text { Intensity (u. a.) } \\
(\text { (x 100) }\end{array}$ & FWHM & $\operatorname{Red}-\chi^{2}\left(\times 10^{-07}\right)$ \\
\hline \multirow{3}{*}{ i) $\mathbf{E} / / \mathbf{a}$} & $7110.80(0.20)$ & $0.21(0.03)$ & & \\
\hline & $7113.36(0.04)$ & $2.00(0.10)$ & $1.98(0.02)$ & 3.3052 \\
\hline & $7114.50(0.03)$ & $3.60(0.10)$ & & \\
\hline \multirow{3}{*}{ i) $\mathbf{E} / / \mathbf{c}$} & $7109.90(0.20)$ & $0.11(0.03)$ & & \\
\hline & $7113.26(0.08)$ & $0.67(0.60)$ & $1.80(0.02)$ & 3.4325 \\
\hline & $7114.74(0.01)$ & $3.44(0.50)$ & & \\
\hline \multirow{5}{*}{ ii) $\mathbf{E} / / \mathbf{a}$} & $7111.60(0.70)$ & $0.70(0.60)$ & $1.91(0.04)$ & \multirow{5}{*}{3.6397} \\
\hline & $7113.48(0.05)$ & $2.10(0.10)$ & $1.91(0.04)$ & \\
\hline & $7114.57(0.04)$ & $3.30(0.20)$ & $1.91(0.04)$ & \\
\hline & 7119.07 (0.07) & $3.00(0.10)$ & $2.60(0.40)$ & \\
\hline & $7121.90(0.10)$ & $11.00(1.00)$ & $5.20(0.80)$ & \\
\hline \multirow{5}{*}{ ii) $\mathbf{E} / / \mathbf{c}$} & $7109.50(0.50)$ & $0.70(0.50)$ & $1.94(0.04)$ & \multirow{5}{*}{3.7702} \\
\hline & $7113.20(0.20)$ & $0.40(0.10)$ & $1.94(0.04)$ & \\
\hline & $7114.76(0.02)$ & $3.40(0.20)$ & $1.94(0.04)$ & \\
\hline & $7119.24(0.01)$ & $9.20(0.30)$ & $2.57(0.02)$ & \\
\hline & $7123.80(0.10)$ & $16.00(1.00)$ & $6.10(0.30)$ & \\
\hline
\end{tabular}

As stated before, in XANES the difference in energy between the peaks of pre-edge corresponds to a direct measurement of the $10 D q$ parameter of the crystalline splitting of the material. However, it is well known that this value is quite different from that obtained by optical spectroscopy, instead it corresponds to about $80 \%$ of the ground state value [33]. In short words, this is mainly because in the final state in a XAS process, the excitation promoted by the X-ray photons let a core hole on the inner shell, as consequence there is a strong influence on the valence band due to the lower screening effect by the nucleus. Regarding $\vec{E} / / \vec{a}$, one observes that the $10 D q$ value obtained from deconvolution of the pre-peak $(\Delta \mathrm{E}=3.7 \mathrm{eV})$ likely corresponds to the optical transition $6 \mathrm{e} \rightarrow 5 \mathrm{a}_{1}(\Delta \mathrm{E}=4.66 \mathrm{eV}$, Fig. 4). Since regular XANES spectroscopy do not provide enough energy resolution (note that the full width at half maximum (FWHM) value is slight below $2 \mathrm{eV}$, Table 1), we do not attempt to attribute the component energy values from pre-edge region to a particular transition in the electronic structure obtained by optical spectroscopy. In an overall scenario, we can point out that, the low-energy pre-edge peak possibly includes $6 e$ and $2 b_{2}$ (or the $t_{2 g}$ group) levels while the highenergy one includes $5 a_{1}$ and $3 b_{1}$ levels ( $e_{g}$ group). The higher transitions (7119 and $7121 \mathrm{eV}$ ) are probably due, respectively, to metal to ligand charge transfer (MLCT) involving 8e $\left(\pi^{*}(\mathrm{CN})\right)[35,36]$ and a long-range order effect [35]. We have performed a 5-peak fitting model in a wider range in energy to include these two higher energy features (B and C in Fig. 2) in 
the pre-peak region (see Table 1). It is worth to note that this long-range fitting does not change the values of intensity and peak position from those obtained from 3-peak model.

$\operatorname{Regarding} \vec{E} / / \vec{c}$, the overall picture is quite similar. We observe, however, a wider distance between $t_{2 g}$ and $e_{g}$ levels $(\Delta \mathrm{E}=4.8 \mathrm{eV})$. Also, the two "edge" peaks (features B and $\mathrm{C}$, on the inset of Fig. 2b) are now resolved in two well separated peaks. It seems quite suggestive that -NO bond, responsible also for low down the crystal symmetry, is in charge for this effect. However, a quantitative analysis of this particular situation requires more experimental details (like a single crystal angular-dependence analysis, for instance) and maybe further theoretical calculations (preferentially using DFT).

$\mathrm{SNP}\left(\left[\mathrm{Fe}(\mathrm{CN})_{5} \mathrm{NO}\right]^{2-}\right.$ anion) exhibits a particular electronic structure, since the lowest unoccupied molecular orbital (LUMO) is the $\pi^{*}(\mathrm{NO})$, which energetically is placed in between the $t_{2 g}$ and $e_{g} 3 d$ iron orbitals [36]. Adopting the nomenclature used elsewhere $[6,11,36]$, the highest occupied molecular orbital (HOMO) is the $d_{x y}$ labelled $2 b_{2}$ (see Fig. 4, left panel), which together with the HOMO-1, the energetically degenerate $d_{x z}$ and $d_{y z}(6 e)$ form the $t_{2 g}$. The e $e_{\mathrm{g}}$ composed of $\mathrm{d}_{\mathrm{z} 2}\left(5 \mathrm{a}_{1}\right)$ and $\mathrm{d}_{\mathrm{x} 2-\mathrm{y} 2}\left(3 \mathrm{~b}_{1}\right)$ constitute the LUMO+1 and LUMO+2 (the energetic order of these two orbitals being inversed between Manoharan and Gray [36], and Delley et al. [6], The 7e $\pi^{*}(\mathrm{NO})$ LUMO has significant admixture of $3 \mathrm{~d}$ orbitals, a characteristic of NO backbonding, which is reflected also in a significant admixture of $\pi^{*}(\mathrm{NO})$ to the $6 e \mathrm{~d}_{\mathrm{xz}}$ and $\mathrm{d}_{\mathrm{yz}}$ orbitals (see e.g. Manoharan and Gray [36], or Braga et al. [37]). Polarized (optical) absorption spectroscopy has allowed for assignment of transitions within the $3 \mathrm{~d}$ manifold and the $\pi^{*}(\mathrm{NO})$, as illustrated in Fig. 4, the lowest lying transition $2 \mathrm{~b}_{2} \rightarrow 7 \mathrm{e}$ is found at $2.5 \mathrm{eV}$, followed by another transition $6 \mathrm{e} \rightarrow 7 \mathrm{e}$ into the LUMO and further intra-d transitions in the energy range of 3.95-4.65 eV. Transitions into higher states have been observed in solution [36], namely the $6 \mathrm{e} \rightarrow 8 \mathrm{e}\left(\pi^{*}(\mathrm{CN})\right)$ at approximately $6.2 \mathrm{eV}$. Ref. [36] provides calculated one-electron MO energies up to much higher levels, including the iron $4 \mathrm{p}$ states and further ligand-based orbitals such as $\pi^{*}(\mathrm{CN}), \sigma(\mathrm{CN})$, and $\sigma(\mathrm{NO})$. Several of these higher states have significant admixture of $3 \mathrm{~d}$, and potential transitions resulting from these calculations would yield energies (with respect to $2 \mathrm{~b}_{2}$ ) around 6.4-8 eV, 9.4-10.1 eV and $14.1 \mathrm{eV}$.
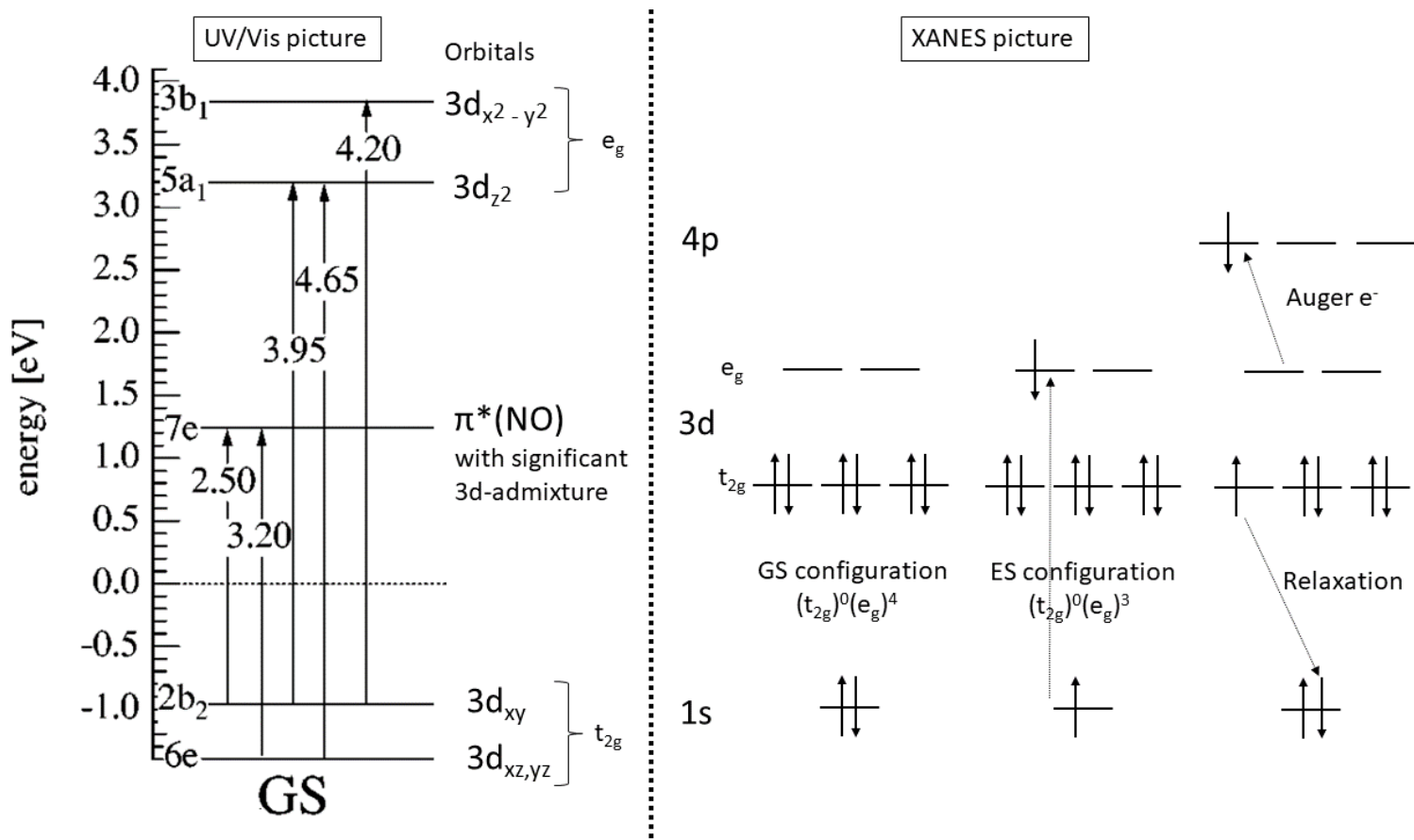
Figure 4: (left) Electronic structure of SNP with optical transitions as observed by polarized absorption spectroscopy [11]; (right) Scheme for the analysis of XANES measurements, assuming an initial transition $1 \mathrm{~s} \rightarrow 3 \mathrm{~d}_{\mathrm{x} 2-\mathrm{y} 2}$ followed by the emission of an Auger electron to $4 \mathrm{p}_{\mathrm{z}}$ and the corresponding relaxation of a t2g electron back to the $1 \mathrm{~s}$.

\subsection{EXAFS results and discussions}
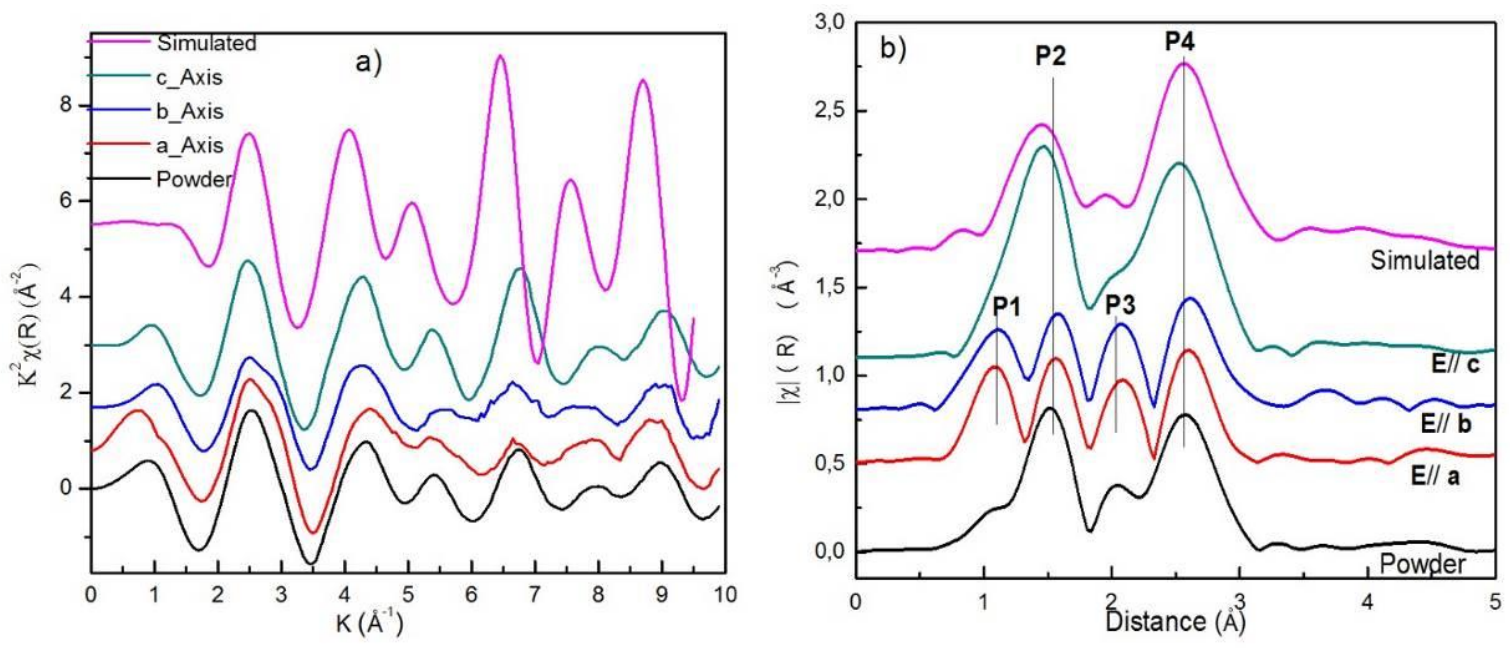

Figure 5: a) Superimposed EXAFS oscillations and b) Fourier Transform functions (using a sine window function as filter) of the SNP experimental data collected for powder, $\vec{E} / / \vec{a}$ (along a-crystal axis), $\vec{E} / / \vec{b}$ (along b-crystal axis), $\vec{E} / / \vec{c}$ (along c-crystal axis) and simulated data without polarization factors. The plots have been done with stack for clarity.

Fig. 5 shows the EXAFS functions $k^{2} \chi(k)$ and the moduli of the Fourier transform (FT) of the experimental data collected for powder, $\vec{E} / / \vec{a}, \vec{E} / / \vec{b}$ and $\vec{E} / / \vec{c}$, and of the simulated data without polarization factors. Overall, the EXAFS spectra for all polarization directions and the powder spectrum exhibit the same main frequency and are in good agreement with the simulated spectrum. However, in the spectra with polarization parallel to the a- and baxis one can clearly observe an additional shoulder at about $3 \AA^{-1}$ as well as some broadening in the range 5.5-6 $\AA^{-1}$. These at first sight rather small differences result in significant differences in real space, as can be seen in Fig. 5b. The peaks are labelled P1, P2, P3 and P4 considering the 0 -distance axis as the reference. In an earlier interpretation made by Gädeke et al. [14], the two strong peaks $\mathrm{P} 2$ and $\mathrm{P} 4$ observed in all spectra would correspond to the $\mathrm{Fe}$ neighboring $\mathrm{C}$ and $\mathrm{N}$ atoms from the $\mathrm{CN}$ ligand. Then, when the polarization vector is parallel to the crystal a- or b- axis, the FT spectra clearly exhibit two more peaks P1 and P3 as a result of the additional scattering contribution from the -NO ligand. Thus, the resulting four peaks would be assigned respectively (according to the authors) to the $\mathrm{N}, \mathrm{C}$ atoms of the $\mathrm{CN}$ ligand and $\mathrm{O}, \mathrm{N}$ of the NO ligand. In the following, we report our finds in EXAFS analysis including multiple scattering. 


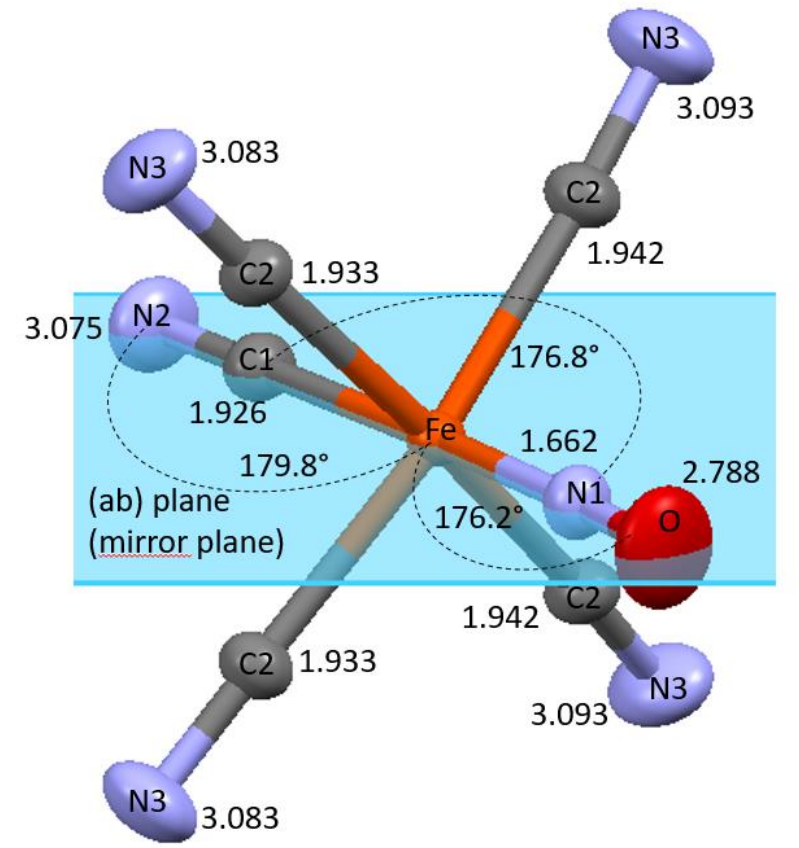

Figure 6: GS configuration according to X-ray diffraction results (at room temperature), highlighting the direction $\mathrm{N}-\mathrm{C}-\mathrm{Fe}-\mathrm{N}-\mathrm{O}$ in the ab plane, with their corresponding bond lengths and angles. The $\mathrm{C}-\mathrm{N}-\mathrm{Fe}-\mathrm{N}-\mathrm{O}$ direction has an angle of approximately $35^{\circ}$ with the a-axis of the crystal.

Fig. 7 shows the magnitude of the experimental functions at Fe K edge of SNP in both, $R$ (the modulus as well as the real part) and $k$ space, superimposed with the curves obtained by a least-square fitting procedure for samples corresponding to the powder, $\vec{E} / / \vec{a}$ and $\vec{E} / / \vec{c}$, respectively. An accurate reproduction of the experimental spectra allowed us to determine the structural parameters characterizing the SNP system. Quantitative results related to the $\mathrm{E}_{0}$ value, interatomic distances $(\mathrm{R})$, coordination numbers $(\mathrm{CNu})$ and Debye-Waller factor $\left(\sigma^{2}\right)$ are presented in Table 2. The amplitude reduction factor $\mathrm{S}_{0}^{2}$ was fixed to 1 for all paths. The structural parameters for the sample $\vec{E} / / \vec{b}$ (not reported) are very close to those for sample $\vec{E} / / \vec{a}$. The goodness of the fit $\left(\mathrm{R}_{\text {factor }}\right)$ is given for each case. The theoretical values of the interatomic distance are also shown for comparison. Differences on FT profile of Fig. 7 and 8 are due to distinct window type used on the transformation, respectively, sine and Hanning.

The powder sample (Fig. 7a, right and left panel) presents two distinct peaks, but it is expected to have contributions from all atoms on each ligand in any axis direction. The blue line on the picture stands for our best fit (green line on the right panel, which corresponds to the real part of the FT function), and the results are given in Table 2. For this fit, due to the fact that we use high-quality single crystals that are well characterized by x-ray diffraction (not shown here), all the coordination numbers were fixed to their nominal value. By doing so, we were able to test the theoretical expected bond distances and compare with the experimental results. Besides the excellent match between theory and experiment, we highlight that the sensitivity of the technique for this system is on the order of one hundredth of an Angströms, which is an important parameter to be considered when dealing with our future experiments dedicated to depict the metastable states. Further, our results also show the slight distortion on the straight angle of the -NO branch with respect to the $a b$-plane, note that direct measurement of Fe-O is slightly lower than the multi-scattering path Fe-N1-O-N1-Fe, respectively, 2.8093(3) and 2.83(1) A. 
When fitting $\vec{E} / / \vec{a}$ (and analogously for $\vec{E} / / \vec{b}$, Figure $7 \mathrm{~b}$, left and right) the direction of the beam polarization with respect to the crystal allows us to see contributions from all the scatters of the system. We expect to see the short Nitrogen bond tied by an oxygen (corresponding to the -NO ligand, lying in the ab-plane) as well as the four-fold degenerated Carbon tied to a longer Nitrogen contribution from the - $\mathrm{CN}$ ligand (out of plane). FEFF calculations have shown the high weight of multiple scattering paths for the total signal, thus the addition of them considerably improved the quality of the fit (Table 2), as previously observed elsewhere [38]. To perform this fit we have considered three different $\mathrm{N}$ bonds on the system, since they are not equivalent: a short one from the -NO ligand (N1), one longer (N2) from - $\mathrm{CN}$, both lying on the mirror plane, and a third from the - $\mathrm{CN}$ ligands that are out of plane (N3, which is four-fold degenerated). It is worth to note that in alternative $\mathrm{N} 2$ and $\mathrm{N} 3$ could be considered as one single contribution, as they are very close in value. However, actually they are not the same, and this is particularly important for our system description since multiscattering paths play an important role for the overall shape of the spectra, as we discussed above. Further, we did try a fit by doing N2 = N3 and we got the same R factor value (0.01), nonetheless the reduced- $\chi^{2}$ favors the model where N2 is independent from N3 (actually, constrained to be twice N2 value). Following this argument, we have also proposed two distinct carbon contributions for our model, one tied to the $-\mathrm{CN}$ ligand in the ab-plane $(\mathrm{C} 1)$ and the other related to carbon from out-of-plane scatterings (C2), which must be four-fold degenerated. On the latter, also the coordination number was constrained to be twice that from c-axis, i.e., $\mathrm{C} 2=4 * \mathrm{C} 1$. As a matter of fact, a model where carbon atoms were fixed to the nominal value gave a far worst fit. On the Table 2, we added a corresponding subscript to make clearer the correspondence among parameter and axis origin. Concerning the bond distances, they are inside the expected range, for instance, N1 matches the value from powder fit as well as the average value from $\mathrm{C} 1$ and $\mathrm{C} 2$ matches the one from powder sample. The coordination numbers of the carbon or nitrogen atoms from -CN ligands are acceptable considering the error bars and the relatively higher values may come from some correlation with other parameters, for instance $\mathrm{C} 1$ has its higher correlation value equals to 0.6737 with $\Delta \mathrm{E}_{0}$. Indeed, $\Delta \mathrm{E}_{0}$ parameter on the a-axis fit is the one that presents the higher error bar comparing to the other fits (even if it is inside the typical range). For sake of clarity, -N1 and $\mathrm{O}$ contributions come from exclusively from -NO ligand and must be always equals to 1. For that reason, we kept fixed coordination numbers of these two components. 

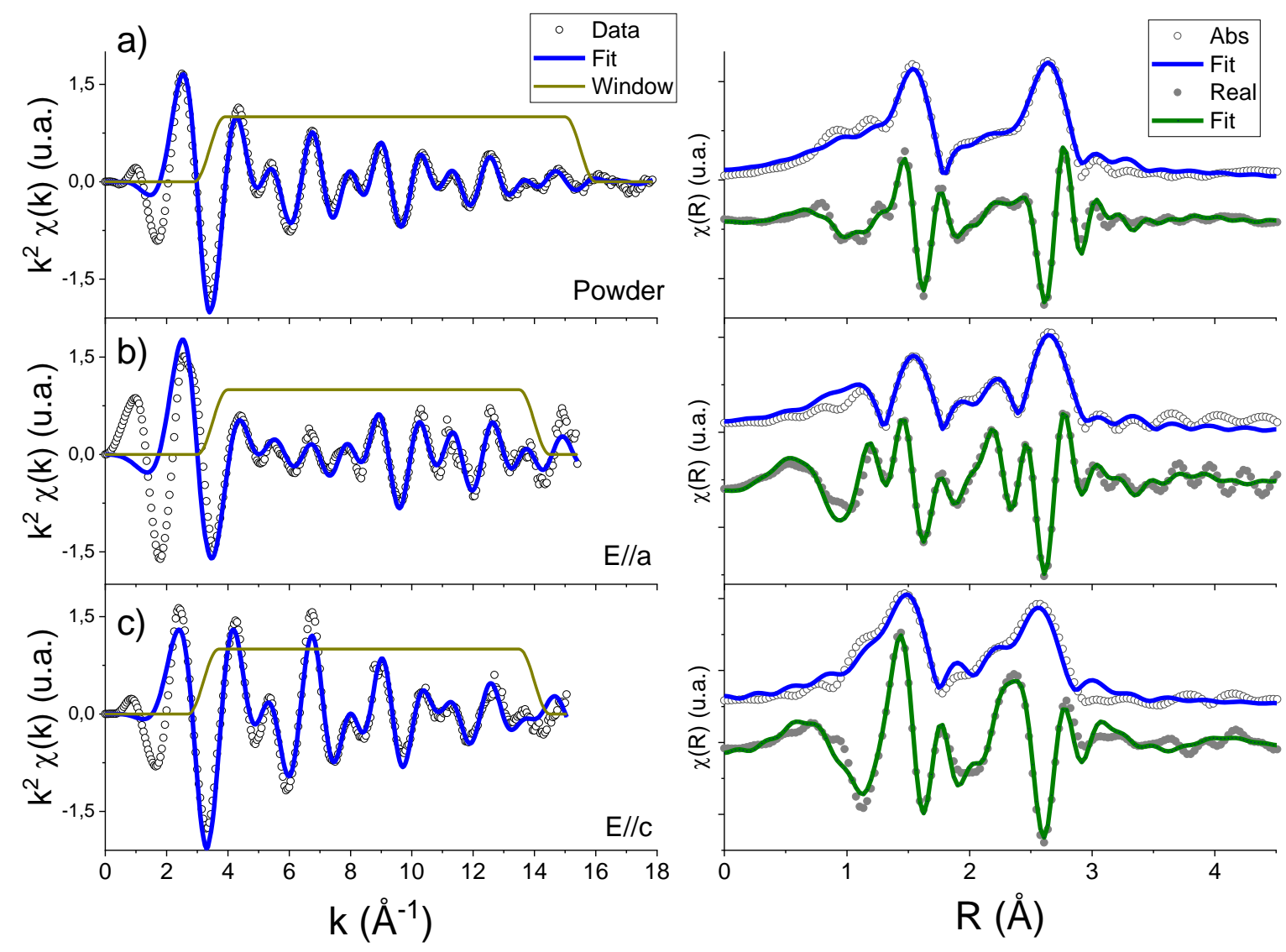

Figure 7. EXAFS signal of Fe K-edge experimental data and the best fit in k-space (left) as well as the Fourier Transform, i.e., in R-space (right) for powder sample (top), $\vec{E} / / \vec{a}$ (middle) and $\vec{E} / / \vec{c}$ (bottom). For the R-plot is shown the magnitude and the real part of the function.

The FT of EXAFS signal in $\vec{E} / / \vec{c}$ (Figure 7c, left and right panels) presents a completely different shape when compared to $\vec{E} / / \vec{a}$ and this can be understood, as already discussed, considering the polarization direction of the beam with respect to the crystal orientation, which acts as a sort of filter for a selected direction. In this particular case, it filters the ab-plane, in which lies the N-C-Fe-N-O branch of the structure. We thus expected to observe only the contributions from the four $-\mathrm{CN}$ ligands out of the ab-plane. Another point to be highlighted is that the apparent similarity of powder and $\vec{E} / / \vec{c}$ FT spectra and any possibility of qualitative misinterpretation based on peak position of intensity is promptly broke when one looks at real part of the spectra (green lines on Fig. 7a and 7c, right panels). The distinction between them means that the origin of the peaks is different, as we actually see on the results of the fits (Table 3), and their close final shape is just a coincidence. 
Table 2. Fitted structural parameters for the Fe bonding atoms of SNP complex. R reff $_{\text {stands }}$ for theoretical interatomic distance. Number in parenthesis are the uncertainties, ${ }^{\mathrm{f}}$ superscript stands for fixed parameter during the fit and the $*$ symbol corresponds to degeneracies of the multi-scattering paths instead of coordination number itself.

\begin{tabular}{|c|c|c|c|c|c|}
\hline Scattering & Ligands & $C N_{u}$ & $\boldsymbol{R}_{\text {reff }}(\AA)$ & $R(\AA)$ & $\sigma^{2}\left(\AA^{2}\right)$ \\
\hline \multicolumn{6}{|c|}{ Powder: $\mathrm{R}_{\text {factor }}=0.01, \Delta \mathrm{E}_{0}=1.6(5) \mathrm{eV}$} \\
\hline$N 1$ & $-\mathrm{NO}$ & $1^{\mathrm{f}}$ & 1.663 & $1.66(1)$ & $0.0041(2)$ \\
\hline$C$ & $-\mathrm{CN}$ & $5^{f}$ & 1.922 & $1.93(1)$ & $0.0041(2)$ \\
\hline$O$ & $-\mathrm{NO}$ & $1^{\mathrm{f}}$ & 2.809 & $2.8093(3)$ & $0.0041(2)$ \\
\hline$N 2$ & $-\mathrm{CN}$ & $5^{f}$ & 3.097 & $3.08(1)$ & $0.0041(2)$ \\
\hline$\sim N 1 \sim O$ & $-\mathrm{NO}$ & $2 *$ & 2.809 & $2.83(1)$ & $0.0041(2)$ \\
\hline$\sim N 1 \sim O \sim N 1$ & $-\mathrm{NO}$ & $1 *$ & 2.810 & $2.83(1)$ & $0.0041(2)$ \\
\hline$\sim C \sim N 2$ & $-\mathrm{CN}$ & $10 *$ & 3.097 & $3.09(2)$ & $0.0041(2)$ \\
\hline$\sim C \sim N 2 \sim C$ & $-\mathrm{CN}$ & $5 *$ & 3.097 & $3.09(2)$ & $0.0041(2)$ \\
\hline \multicolumn{6}{|c|}{$\vec{E} / / \vec{a}: \mathrm{R}_{\text {factor }}=0.01, \Delta \mathrm{E}_{0}=-2(1) \mathrm{eV}$} \\
\hline$N 1$ & $-\mathrm{NO}$ & $1^{\mathrm{f}}$ & 1.663 & $1.64(1)$ & $0.0032(6)$ \\
\hline$C 1$ & $-\mathrm{CN}_{\mathrm{c}}$ & $1.2(2)$ & 1.913 & $1.880(9)$ & $0.0032(6)$ \\
\hline$C 2$ & $-\mathrm{CN}_{\mathrm{ab}}$ & $4.8(8)$ & 1.922 & $1.96(2)$ & $0.0032(6)$ \\
\hline$O$ & $-\mathrm{NO}$ & $1^{\mathrm{f}}$ & 2.809 & $2.82(1)$ & $0.0032(6)$ \\
\hline$N 2$ & $-\mathrm{CN}_{\mathrm{c}}$ & $1.2(2)$ & 3.085 & $3.089(9)$ & $0.0032(6)$ \\
\hline$N 3$ & $-\mathrm{CN}_{\mathrm{ab}}$ & $4.8(8)$ & 3.096 & $3.11(2)$ & $0.0032(6)$ \\
\hline$\sim N 1 \sim O$ & $-\mathrm{NO}$ & $2 *$ & 2.809 & $2.81(2)$ & $0.0032(6)$ \\
\hline$\sim N 1 \sim O \sim N 1$ & $-\mathrm{NO}$ & $1 *$ & 2.810 & $2.81(2)$ & $0.0032(6)$ \\
\hline$\sim C 1 \sim N 2$ & $-\mathrm{CN}_{\mathrm{c}}$ & $4.8(8)^{*}$ & 3.085 & $3.05(1)$ & $0.0032(6)$ \\
\hline$\sim C 1 \sim N 2 \sim C 1$ & $-\mathrm{CN}_{\mathrm{c}}$ & $1.2(2)^{*}$ & 3.085 & $3.05(1)$ & $0.0032(6)$ \\
\hline$\sim C 2 \sim N 3$ & $-\mathrm{CN}_{\mathrm{ab}}$ & $4.8(8)^{*}$ & 3.096 & $3.15(4)$ & $0.0032(6)$ \\
\hline \multicolumn{6}{|c|}{$\vec{E} / / \vec{c}: \mathrm{R}_{\text {factor }}=0.02, \Delta \mathrm{E}_{0}=0.1(8) \mathrm{eV}$} \\
\hline$C$ & $-\mathrm{CN}_{\mathrm{ab}}$ & $4.6(4)$ & 1.922 & $1.930(7)$ & $0.0015(5)$ \\
\hline$N$ & $-\mathrm{CN}_{\mathrm{ab}}$ & $4.6(4)$ & 3.097 & $3.092(8)$ & $0.0015(5)$ \\
\hline$\sim C \sim N$ & $-\mathrm{CN}_{\mathrm{ab}}$ & $9.2(8) f^{\mathrm{f}} *$ & 3.097 & $3.10(1)$ & $0.003(1)$ \\
\hline$\sim C \sim N \sim C$ & $-\mathrm{CN}_{\mathrm{ab}}$ & $4.6(4)^{\mathrm{f} *}$ & 3.097 & $3.10(2)$ & $0.003(1)$ \\
\hline
\end{tabular}

We have seen so far with the EXAFS fitting of the different faces of the crystal that multi-scattering paths have a huge contribution for the final aspect of the curve and they cannot 
be neglected during the modeling. To make clearer this issue, Fig. 8 highlight the individual contributions of the scattering paths of the model to the fit of the powder sample, as an example of the importance of such scatterings for the overall spectrum. In the left panel is drawn the FT of EXAFS in R-space (open circles), its fit (blue line) and the individual contribution of each scattering to the fit (actually, the real part). It is shown clearly that even if we have 4 different atomic contributions (short $\mathrm{N}$ and $\mathrm{C}$ atoms from - $\mathrm{CN}$ ligand as well as $\mathrm{O}, \mathrm{N}$ from NO ligand) in the structure, they cannot be individualized as a single peak in the FT (nor even a convolution), which is a current misconception in EXAFS analysis. On a hypothetical radial distribution function scenario, carbon should count as the position and intensity of the first high FT peak $(\sim 1.6 \AA$, without phase correction), but definitely both nitrogen scatterings contribute for this first peak as well as multiple-scatterings, which spans from 0.5 up to $3.5 \AA$. In fact, their presence dominates the high term (R > $2 \AA$ ) on FT of both data. Actually, for longer distances, the focusing effect promoted by the alignment of the scatters highlights the longer contributions, as viewed in the intensities of the back transformed waves on right panels of Fig. 8. It is worth to note that the presence of simulated multi-scattering paths contributes to reinforce the stability of the proposed model and to support the veracity of the fit results.

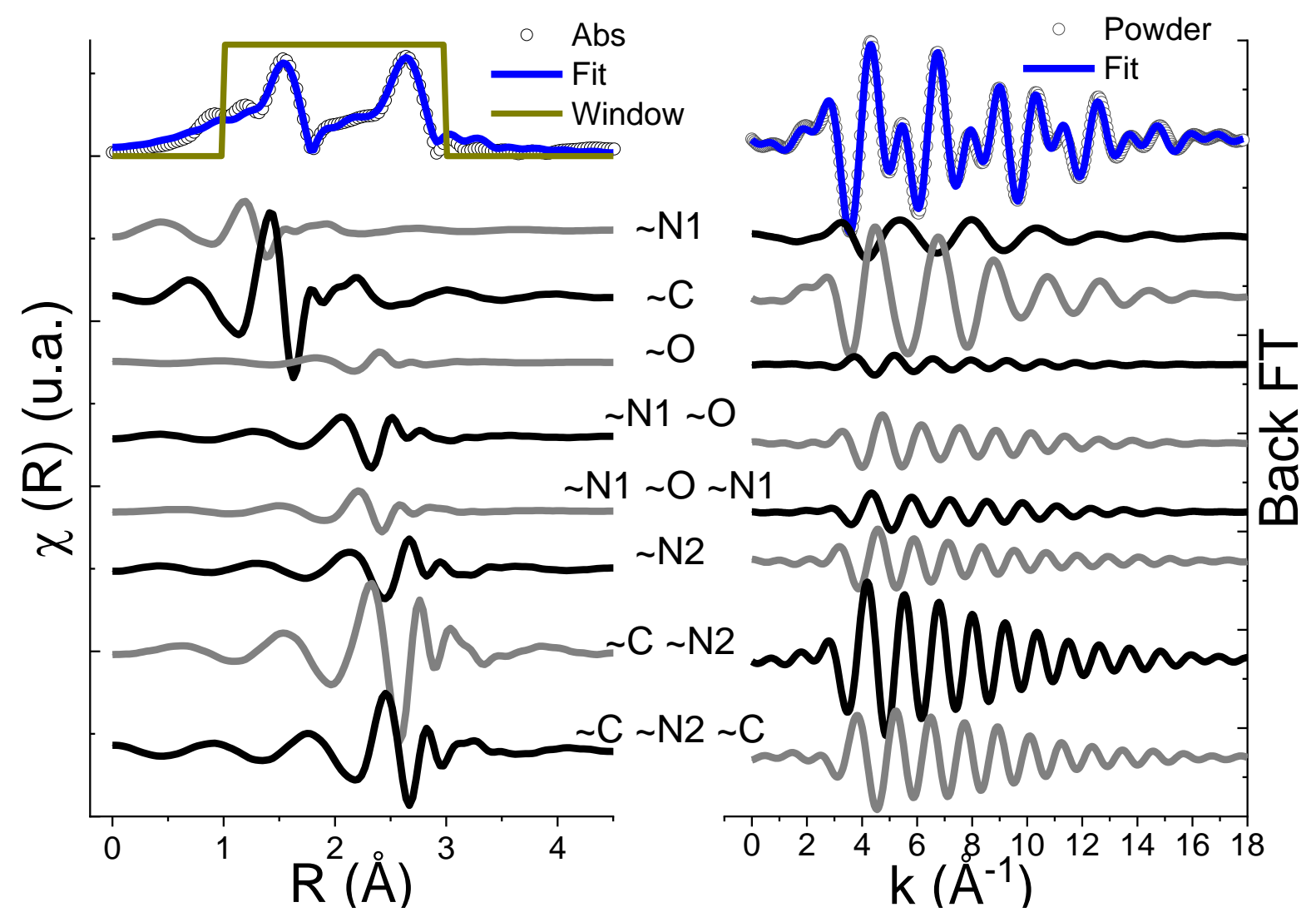

Figure 8. Individual contribution of the scattering paths for the real part of the FT EXAFS signal (left) as well as the back Fourier Transform (right) from $\mathrm{Fe}$ K-edge of SNP powder sample.

Summarily, our EXAFS results show great accordance with the expected structure and we were able to describe fine aspects from the crystal, such as the distinction of 3 different $\mathrm{N}$ contributions on $\vec{E} / / \vec{a}$ fit. On the other hand, this full description with a particular importance of the inclusion of multi-scattering peaks and their influence for the final aspect of the Fourier 
Transform of the EXAFS is fundamental to update the interpretation given by Gadeke et al. [14], which attributed single peaks to single atomic contributions. As our analysis clearly shows this oversimplified interpretation is inadequate and might even lead to false conclusions if taken as such.

\section{Conclusion}

To conclude, we have shown that the properties of a complex system such as SNP $\left(\mathrm{Na} 2\left[\mathrm{Fe}(\mathrm{CN})_{5} \mathrm{NO}\right] 2 \mathrm{H}_{2} \mathrm{O}\right.$, prototype) can be investigated through polarized $\mathrm{x}$-ray absorption spectroscopy. Fine and full modelling of XANES and EXAFS allowed to see details from the lower symmetry of the crystal (e.g., difference in pre-peak XANES and coordination numbers after EXAFS fitting). FEFF.9 code fails to nicely describe minor, nonetheless important, XANES features, however it is suitable to simulate polarization dependence in the EXAFS regime. In the XANES region, SNP exhibits pre-edge features which correspond to the combination of $1 s \rightarrow 4 p$ and $1 s \rightarrow 3 d$ transitions with a dominant character assimilated to a dipole character, as revealed by the difference between the two axes. A deconvolution of $\mathrm{Fe}$ pre-peaks allows to see that according to the energy range, they can possibly include $6 e$ and $2 b_{2}$ levels of $t_{2 g}$ group and $5 a_{1}$ and $3 b_{1}$ of $e_{g}$ group. Another possibility is the presence of $8 \mathrm{e}$ $\left(\pi^{*}(\mathrm{CN})\right.$ at high pre-peak energy. Even if a perfect correspondence with optical transition was not possible, our results pave a direction in how to perform further investigations coupling well-designed XAS study (using angle dependence detection and low temperature), FEFF simulation and optical experiments to solve the electronic structure of the crystal and to study its metastable state. EXAFS showed good agreement with theoretical values and we were able to also observe the influence of the distortion of the structure on coordination number and bond distances. Nevertheless, the most important achievement was to update the old (mis)interpretation in literature in which associated single peaks on Fourier transform to single atomic contributions of the crystal. We have seen the fundamental role played by multiscattering paths to the overall shape of the spectra and we expect that this work contributes to overcome such aged, guileless but still present understanding of the EXAFS as a radial distribution function.

\section{Acknowledgments}

The authors are grateful to IUPAP-IUCr-LAAMP within the ICSU Grants Programme 20162019 (Ref: 41100IUP12) who provided the support to Duclair Tchana Kamgne and Bridinette Thiodjio Sendja for the training in ELETTRA Synchrotron. They are also grateful to the XAFS beamline staff (Dr Simone Pollastri, Mr Luca Olivi and Mr Ricardo Grisonich) of Elettra Sincrotrone (Trieste, Italy). They also extend their acknowledgement to Andrea Lausi responsible of the LAAAMP in ELETTRA and Vivian Zaccaria of ICTP external of office activities. We thank Theo Woike for preparation of oriented SNP single crystals.

\section{References}

[1] M. D. Carlson, P. M. Eckmann, "Review of Vasodilators in acute decompensated heart failure: the old and the new", Journal of Cardiac Failure 19, 478-493 (2013). 
[2] S. Huerta, S. Chilka, B. Buenavida, "Nitric Oxide Donors : Novel Cancer Therapeutics" International Journal of Oncology 33, 909-927 (2008).

[3] P. Gütlich, Y. Garcia, T. Woike, "Photoswitchable coordination compounds", Coord. Chem. Rev. 219-221, 839-879 (2001).

[4] P. Coppens, I. Novozhilva, A. Kovalevsky, "Photoinduced Linkage Isomers of TransitionMetal Nitrosyl Compounds and Related Complexes", Chem. Rev. 102, 861-883 (2002).

[5] M. D. Carducci, M. R. Pressprich, P. Coppens, "Diffraction studies of photoexcited crystals: Metastable nitrosyl-linkage isomers of sodium nitroprusside", J. Am. Chem. Soc. 119, 26692678 (1997).

[6] B. Delley, J. Schefer, Th. Woike, "Giant lifetimes of optically excited states and the elusive structure of sodiumnitroprusside.” J. Chem. Phys. 107, 10067-10074 (1997).

[7] D. Schaniel, Th. Woike, J. Schefer, V. Petříček, "Structure of the light-induced metastable state SII in $\mathrm{Na}_{2}\left[\mathrm{Fe}(\mathrm{CN})_{5} \mathrm{NO}\right] \cdot 2 \mathrm{H}_{2} \mathrm{O}$ ”, Phys. Rev. B 71, 174112 (2005).

[8] D. Schaniel, Th. Woike, J. Schefer, V. Petříček, K. W. Krämer, H. U. Güdel, "Neutron diffraction shows a photoinduced isonitrosyl linkage isomer in the metastable state SI of $\mathrm{Na}_{2}\left[\mathrm{Fe}(\mathrm{CN})_{5} \mathrm{NO}\right] \cdot 2 \mathrm{D}_{2} \mathrm{O}$ ”. Phys. Rev. B 73, 174108 (2006).

[9] D. Schaniel, M. Nicoul, Th. Woike, "Ultrafast reversible ligand isomerization in $\mathrm{Na}_{2}\left[\mathrm{Fe}(\mathrm{CN})_{5} \mathrm{NO}\right] \cdot 2 \mathrm{H}_{2} \mathrm{O}$ single crystals”, Phys. Chem. Chem. Phys. 12, 9029-9033 (2010).

[10] G. Galle, M. Nicoul, Th. Woike, D. Schaniel, E. Freysz "Unraveling the mechanism of NO ligand photoisomerism by time-resolved infrared spectroscopy", Chem. Phys. Lett. 552, 64-68 (2012).

[11] D. Schaniel, J. Schefer, B. Delley, M. Imlau, Th Woike, "Light-induced absorption changes by excitation of metastable states in $\mathrm{Na}_{2}\left[\mathrm{Fe}(\mathrm{CN})_{5} \mathrm{NO}\right] \cdot 2 \mathrm{H}_{2} \mathrm{O}$ single crystals ", Phys. Rev. B 66, 085103 (2002).

[12] D. Schaniel, M. Imlau T. Weisemoeller T. Woike K. W. Krämer H.-U. Güdel "Photoinduced Nitrosyl Linkage Isomers Uncover a Variety of Unconventional Photorefractive Media", Adv. Mater. 19, 723-727 (2007). 
[13] T. Woike, W. Krasser, H. Zöllner, W. Kirchner, S. Haussühl, Z. "Population dynamics of the two light-induced metastable states in $\mathrm{Na} 2\left[\mathrm{Fe}(\mathrm{CN})_{5} \mathrm{NO}\right] \cdot 2 \mathrm{H}_{2} \mathrm{O}$ single crystals" $\mathrm{Z}$. Phys. D 25, 351-356 (1993).

[14] W. Gadeke, E. E. Koch, G. Drager, R. Frahm and V. Saile; "Electronic structure and local geometry of $\mathrm{Na}\left[\mathrm{Fe}(\mathrm{CN})_{5} \mathrm{NO}\right] \cdot 2 \mathrm{H}_{2} \mathrm{O}$ single crystals investigated by polarizationdependent x-ray absorption spectroscopy;" Chem. Phys. 124 (1988) 113-1 19.

[15] S. Nanba, D Asakura, M. Okubo, H. Zhou K. Amemiya, K. Okada, Per-Anders Glans Catherine A . Jenkins, Elke Arenholz and J. Guo "Anisotropic charge-transfer effects in the asymmetric $\mathrm{Fe}(\mathrm{CN})_{5} \mathrm{NO}$ octahedron of sodium nitroprusside: a soft X-ray absorption spectroscopy study" Phys. Chem. Chem. Phys. 16, 7031-7036 (2014).

[16] S.Yamazaki, T. Matsui, T.Sato, Y. Arita, T.Nagasaki, "EXAFS study of reduced ceria doped with lanthanide oxides", Solid State Ionics 154, 113-118 (2002).

[17] H. Deguchi, H. Yoshida, T. Inagaki, M. Horiuchi, "EXAFS study of doped ceria using multiple data set fit”, Solid State Ionics 176, 1817 - 1825 (2009).

[18] D. Cabaret, A. Bordage, A. Juhin, M. Arfaoui, E. Gaudry "First-principles calculations of $X$-ray absorption spectra at the $K$ edge of $3 d$ transition metals: an electronic structure analysis of the pre-edge". Phys. Chem. Chem. Phys. 12, 5619-5633 (2010).

[19] O. Lobacheva, M. Chavarha, Y.M. Yiu, T.K. Sham, L.V. Goncharova. "The local structure and ferromagnetism in Fe-implanted $\mathrm{SrTiO}_{3}$ single crystals" J. Appl. Phys. 116, 013901 (2014).

[20] F. Giannici, G. Gregori, C.Aliotta, A. Longo, J.Maier, A. Martorana, "Structure and Oxide Ion Conductivity: Local Order, Defect Interactions and Grain Boundary Effects in AcceptorDopedCeria”. Chem. Mater. 26 5994-6006 (2014).

[21] F. Bottomley, P. S. White, "Redetermination of the structure of disodium pentacyanonitrosylferrate (sodium nitroprusside)” Acta Cryst. B35, 2193-2195 (1979).

[22] P. T. Manoharan, W. C. Hamilton, “Crystal structure of sodium nitroprusside”, Inorg. Chem. 2, 1043-1047 (1963).

[23] A. Di. Cicco, G. Aquilanti, M. Minicucci, E. Principi, N. Novello, A Cognigni, L. Olivi, "Novel XAFS capabilities at ELETTRA synchrotron light source" J. Phys.: Conf. Ser. 190, 012043 (2009). 
[24] B. Ravel, M. Newville, "Athena, Artemis, Hephaestus: data analysis for X-ray absorption spectroscopy using Ifeffit" $j$. Synchrotron rad. 12 (2005a) 537-41. https://doi.org/10.1107/s0909049505012719.

[25] T. Hashimoto, A.Yoshiasa, M. Sugahara, H. Arima, H. Fukui, K-I. Murai, M. Okube, "High Energy Ferrite Magnets" J. Phys.: Conf. Ser. 121 (2008) 102002. https://hal.archivesouvertes.fr/jpa-00254763.

[26] B. Thiodjio Sendja, D. Tchana Kamgne, G. Aquilanti, L. Olivi, J. R. Plaisier. "Lowrange thermal investigation of zincblende-type ZnS by combined extended X-ray absorption fine structure and X-ray diffraction techniques". Physica B: Condensed Matter 545 (2018) 481-490. https://doi.org/10.1016/j.physb.2018.06.005.

[27] J. J. Rehr, J. J. Kas, F. D. Vila, M. P. Prange, K. Jorissen 'Parameter-free calculations of X-ray spectra with FEFF9 “'Phys. Chem. Chem. Phys.12 (2010) 5503-5513, https://doi.org/10.1039/B926434E.

[28] M. Roy and S. J. Gurman, "An investigation of the use of the Hedin+Lundqvist exchange and correlation potential in EXAFS data analysis", J. Synchrotron Rad. 8 (2001) 1095-1102 DOI: $10.1107 /$ S0909049501006434.

[29] M. Muñoz, O.Vidal, C.Marcaillou, S. Pascarelli, O. Mathon, and F. Farges, "Iron oxidation state in phyllosilicate single crystals using Fe-K pre-edge and XANES spectroscopy: Effects of the linear polarization of the synchrotron X-ray beam", American Mineralogist 98 (2013) 1187-1197, https://doi.org/10.2138/am.2013.4289 .

[30] G. Waychunas, M. Apted, Jr G. Brown "X-ray K-edge absorption spectra of Fe minerals and model compounds: near edge structure". Physics and Chemistry of Minerals 10 (1983) 19. https://doi.org/10.1007/BF01204319.

[31] A. Berry, O’Neill HSTC, K. Jayasuriya, S. Campbell, G. Foran "XANES calibrations for the oxidation state of iron in a silicate glass". American Mineralogist 88 (2008) 967-977. http://repository.kln.ac.lk/handle/123456789/4096. 
[32] V. Vercamer, O. J. Y. Myrtille, G. Hunault, M. Lelong, W. Haverkort, G. Calas, A. Yusuke, H. Hijiya, L. Paulatto, C. Brouder, M.-A. Arrio, and A. Juhi ' Calculation of optical and k-pre-edge absorption spectra for ferrous iron of distorted sites in oxide crystals" Phys. Rev. B 94, (2016) 245115. DOI:https://doi.org/10.1103/PhysRevB.94.245115.

[33] G. Dräger, R. Frahm, G. Materlik, O. Brümmer, ' 'On the Multipole Character of the XRay Transitions in the Pre-Edge Structure of Fe K Absorption Spectra. An Experimental Study”Phys. Stat. Sol. B 146 (1988) 287. https://doi.org/10.1002/pssb.2221460130.

[34] T. E. Westre, P. Kennepohl, J. G. DeWitt, B. Hedman, K.O. Hodgson, and E. I. Solomon. 'A Multiplet Analysis of Fe K-Edge 1s $\rightarrow 3 d$ Pre-Edge Features of Iron Complexes'J. Am. Chem. Soc. 119 (1997) 6297. https://doi.org/10.1021/ja964352a.

[35] T. J. Penfold , M. Reinhard, M. H. Rittmann-Frank,I. Tavernelli, U. Rothlisberger, C. J. Milne, P. Glatzel, and M. Chergui. ' X-ray Spectroscopic Study of Solvent Effects on the Ferrous and Ferric Hexacyanide Anions"' J. Phys. Chem. A 118 (2014) 9411. https://doi.org/10.1021/jp5055588.

[36] T. Manoharan, H.Gray, "Electronic Structure of Nitroprusside Ion” J. Am. Chem. Soc. 87 (1965) 3340-3348. https://doi.org/10.1021/ja01093a011.

[37] M. Braga, A. C. Pavao, J. R. Leite, 'The Use of Imaging Plates in Electron-Density Mapping with Synchrotron X-rays", Phys. Rev. B 23 (1981) 4328-4336. doi.org/10.1107/S0909049595006170.

[38] G Tolkiehn, P. Rabe, A. Werner 'experimental investigation of the systematic $k$-and $z$ dependence of exafs back-scattering amplitudes and comparison with theory' 'Institut fur Experimentalphysik, Universitat Kiel Olshausenstr in 'Inner-Shell and X-Ray Physics of Atoms and Solids" 40-60 pp. 675-678 (1981) D-2300 Kiel. 